\title{
Value of Two-Dimensional Speckle Tracking at Rest and During Dobutmaine Stress Echocardiography in Diagnosis of Coronary Artery Disease
}

\author{
Ahmed M. Saleh ( $\square$ salehcardio@hotmail.com ) \\ Stadtisches Klinikum Braunschweig gGmbH https://orcid.org/0000-0002-7420-6891 \\ Konstantin Zintl \\ Klinikum Coburg GmbH \\ Yamen Mohrez \\ Klinikum Coburg GmbH \\ Johannes Brachmann \\ Klinikum Coburg GmbH
}

\section{Research}

Keywords: Speckle Tracking, Echocardiography, Dobutmaine Stress

Posted Date: January 23rd, 2021

DOI: https://doi.org/10.21203/rs.3.rs-149120/v1

License: (9) This work is licensed under a Creative Commons Attribution 4.0 International License. Read Full License 


\section{Abstract}

Introduction

CAD (coronary artery disease) is a major cause of death and disability in developed countries. Exercise testing is recommended as a first-line diagnostic test in patients with stable angina pectoris. However, the limited sensitivity and specificity have been criticized. Myocardial strain can predict the presence of significant coronary artery disease.

Aim of work

to assess the utility of myocardial strain obtained by 2D-speckle tracking at rest and under stress for the detection of $C A D$ in patients referred to chest pain unit with stable and low risk unstable angina pectoris.

Methods

108 individuals matched the inclusion criteria and gave their written consent to be enrolled in this study. Coronary angiography was performed within 48 hours from admission to the chest pain unit. Myocardial strain by 2D-speckle tracking was obtained at rest and under dobutamine stress.

Results

Global longitudinal strain at stress revealed a moderate correlation with the presence of significant CAD; $r=0.41, p<0.0001$. While a moderate correlation was established between global strain under stress with the severity of coronary occlusion, $r=0.62, p<0.0001$. Global longitudinal strain under stress showed a sensitivity of $74.1 \%$ and a specificity of $76.7 \%$ in detecting significant CAD at a cut-off value of -19.1 .

Conclusion

Longitudinal strain parameters at rest and under stress could predict the presence of coronary artery disease in patients with stable angina pectoris. The obtained strain data could add value in detecting the severity of CAD.

\section{Introduction}

Coronary artery disease is characterized by atherosclerosis in the epicardial coronary arteries. The reduction in coronary artery flow may be symptomatic or asymptomatic, occurs with exertion or at rest and culminate in myocardial infarction or angina, depending on the severity of involved coronary obstruction and the rapidity of development. ${ }^{(1)}$

Dobutamine stress echocardiography (DSE) is one of the established tools for diagnosing coronary artery disease (CAD). However, visual diagnosis of wall motion abnormalities during DSE lacks high accuracy due to relatively low interobserver agreement depending on the qualitative nature of diagnosis and its reliance on the expertise of the cardiologist. $(2,3)$ 
Despite efforts to standardize DES by using wall motion scores and enhance myocardial border detection with transpulmonary contrast, the learning curve is steep and variable reproducibility between observers and institutions is still observed. $(4,5)$

Measurements of myocardial global longitudinal strain and strain rate (GLS) are newer indices that have the potential to overcome these limitations. Echocardiographic longitudinal strain reliably measures regional myocardial deformation and deformation rate (strain rate, SR). Left ventricular wall motion is depicted accurately at rest and during acute and chronic ischemia, including stress-induced ischemia in animal models. Recent reports on the use of myocardial strain during DSE for viability and ischemia are promising. $(6,7,8,9)$

We focused in this study on evaluating the utility of myocardial strain at rest and under stress obtained during dobutamine Echocardiography in relation to the invasive coronary angiography results in patients presenting with acute chest pain.

\section{Methods}

Patients with typical chest pain were admitted to the chest pain unit (CPU) at Coburg hospital, Germany between February 2019 and August 2020. Three hundred ten patients with stable and unstable angina (TIMI risk score 0-1) were screened. Among them 108 individuals matched the inclusion criteria and gave their written consent to be enrolled in this study (Figure 1). All included subjects were older than 18 years and suited for stress testing. Coronary angiography was performed within 48 hours from admission to the chest pain unit. For the purposes of this analysis, we characterized significant coronary stenosis as $\geq$ $70 \%$ luminal obstruction. Although less severe stenosis might be associated with risk for cardiovascular events, we elected to use a widely accepted standard for defining angiographic significance. Exclusion criteria were elevated troponin level, ST-segment elevation or depression as well as dynamic ECG-changes during admission at $\mathrm{CPU}$, history of coronary artery disease or acute myocardial infarction, coronary artery bypass grafting, chronic total coronary occlusion, significant valvular heart disease, end stage renal failure, or refusal to give the written consent.

After enrollment, Dobutamine stress echocardiography was conducted before invasive assessment with coronary angiography.

\section{Echocardiography}

Examinations were performed with a digital ultrasonic device system (Vivid 9, GE Vingmed Ultrasound, Horten, Norway). In harmonic mode 2.0/4.3 MHz with maximal frame per second (FPS) count available at necessary sector width. Range of FPS was from 64 to 112 with mean value 83.

Conventional echocardiography study was done including 2D based M-mode measures of cardiac chambers and Ejection Fraction (EF\%), pulsed and continuous wave Doppler studies, Color Doppler study, calculation of ejection fraction by Simpson`s method and analysis of wall motion abnormalities. 
DSE was performed using a standard staged protocol. Dobutamine was infused through a peripheral infusion line intravenously with a mechanical pump starting at dose of $10 \mathrm{mg} / \mathrm{kg} / \mathrm{min}$. The dose was increased at 3-min intervals to 20,30 , and $40 \mathrm{mg} / \mathrm{kg} / \mathrm{min}$ with intravenous atropine up to $2 \mathrm{mg}$ given, if necessary, to augment the heart rate response. Blood pressure and electrocardiogram were monitored continuously. Criteria for terminating the test were achieving a target heart rate response of $85 \%$ of the age-predicted maximum, development of wall motion abnormality, angina pectoris, severe ischemic electrocardiographic changes, systolic blood pressure $>240 \mathrm{~mm} \mathrm{Hg}$, abnormal blood pressure reaction during stress, or significant arrhythmia.

Images were obtained by two experienced echocardiographers with patients in the lateral decubitus position. Standard 2D grayscale images of three standard apical views (four-chamber, two-chamber, and apical long-axis) and parasternal long-axis and parasternal short-axis views at the level of mitral valve, papillary muscles, and apex were acquired at rest, at a dobutamine dose of $20 \mathrm{mg} / \mathrm{kg} / \mathrm{min}$, at peak stress, and at recovery $1 \mathrm{~min}$ after stress. Per protocol, a cine image of one representative cardiac cycle per stage and view was digitally stored for later offline analysis. To optimize speckle-tracking at high heart rate, images were individually optimized for left ventricular analysis, and the frame rate was increased to achieve at target of 60 to $90 \mathrm{frames} / \mathrm{sec}$ without compromising endocardial border detection. Wall motion was analyzed visually by two experienced echocardiographers blinded to the results of other investigations. The operators repeated the evaluation of wall motion abnormalities of 20 random studies at the end of enrollment to assess intra- and interobserver variation.

Quantification of strain measurements

Echocardiographic images were obtained prior to coronary angiography. Three uninterrupted cardiac cycles were applied for each of three standard apical (two-, three-, and four-chamber) views and were kept for off-line longitudinal strain analysis, using EchoPAC software (GE Ultrasound). For assessment of longitudinal strain, we recorded standard 2D ultrasound images with a frame rate between 60 and 90 frames per second (fps) from the standard views. The endocardium was manually marked out from selected cineloops of apical view images. A further manual adjustment of the region of interest was applied after visual evaluation. The Full image was excluded if $\geq 2$ segments were poorly tracked. Speckle tracking was carried out on all three apical views of rest images and negative global systolic longitudinal strain was estimated. The average of GLS in apical 4, 3, and 2 chamber imaged was used for our analysis.

Statistical Analysis

All statistical analyses were performed using SPSS 21.0 (SPSS Inc, Chicago, Illinois). Continuous variables are expressed as mean \pm standard deviation and categorical variables are expressed as counts and \%. Diagnostic measures including sensitivity, specificity, positive predictive value (PPV), and negative predictive value (NPV) were calculated. The receiver operating characteristic (ROC) curve analyses were 
performed to compare the diagnostic performance of myocardial strain at rest and under stress. Pearson's correlation was used to assess the strength of association among variables. Inter- and intraobserver reproducibility values were evaluated through the intra-class correlation coefficient (ICC). For all analyses, $p<0.05$ was considered to be statistically significant.

\section{Results}

We examined 108 patients presented with stable and unstable angina pectoris at the chest pain unit to whom coronary angiography was performed. The diagnosis was based on the clinical presentation, ECG results and laboratory findings. Subjected who were excluded from the study are identified in Figure 1. The patients were subjected to dobutamine stress echocardiography prior to the invasive evaluation to determine the global longitudinal strain values (Figure 2).

Demographic features

Women accounted for $50 \%$ of our study cohort (No. 54). Eighty-seven patients were hypertensive while Fifty-two were already diagnosed with diabetes before admission. Forty-one of the studied group were smokers (38\%) and forty patients showed either elevated lipid profile or were taking lipid-lowering drugs. (Table 1). The mean age in our study group was 64 years old +10 and mean Body mass index was 25.4 $\mathrm{kg} / \mathrm{m}^{2}+3.8$. Troponin levels at admission were $0.0 .081 \mathrm{ng} / \mathrm{ml}+0.03$. Thirty-five subjects of our cohort showed a significant coronary lesion $>70 \%$ in coronary angiography.

\section{Echocardiographic results}

We detected segmental wall motion abnormalities (WMA) under dobutamine stress (in at least one segment) in 31 patients. Global longitudinal strain (GLS) at rest was significantly lower in patients with CAD compared to patients without $(-16.9 \% \pm 2.8$ vs. $-18.4 \% \pm 3.1$; $p$-value $<0.001)$. Likewise, GLS under stress was lower in subjects with significant CAD $(-20.8 \% \pm 3.3$ vs. $-21.9 \% \pm 3.7)$ (Table. 1$)$

A weak correlation between WMA under stress and the presence of CAD was established, $r=0.26, p$ $<0.05$. No correlation between WMA under stress and the severity of coronary lesion was found, $p>0.05$. Global longitudinal strain at rest obtained by speckle tracking showed a moderate correlation with the presence of significant CAD; $r=0.36, p<0.05$. Global strain at stress revealed a moderate correlation with the presence of significant CAD as well; $r=0.41, p<0.0001$. Moreover, a moderate correlation was found between global strain at rest and the angiographic severity of coronary lesion (in \%), $r=0.53, p<0.0001$ (Figure 3) while a moderate correlation was established between global strain under stress with the severity of coronary occlusion, $r=0.62, p<0.0001$ (Figure 4).

In a one vessel CAD a mean global strain of $-18.2 \% \pm 2.3$ was detected at rest and a mean global strain of $-22.3 \% \pm 2.9$ was detected under stress. The patients who showed angiographic evidence of 2-vessel CAD revealed a mean global strain at rest of $-15.4 \% \pm 1.5$ and a mean global strain under stress of $-17.7 \% \pm 2.4$. 
Patients with 3-vessel coronary disease involvement showed a mean global strain of $-13.3 \% \pm 1.1$ at rest and of $-14.6 \% \pm 1.6$ under stress. (Figure 5 )

Global longitudinal strain under stress showed a sensitivity of $74.1 \%$ and a specificity of $76.7 \%$ in detecting significant CAD at a cut-off value of -19.1 (AUC: 0.754 ) with a positive predictive value of $56.1 \%$ and a negative predictive value of $88.06 \%$. (Figure 6, Table 2 )

Reproducibility of obtained parameters

Inter-observer agreement tested by ICC was 0.84 for GLS at rest, 0.83 for GLS under stress, and 0.74 for WMA under stress. We calculated an intra-observer agreement of $0.85,0.87$ and 0.79 for the same values respectively.

\section{Discussion}

The present study explored the changes of myocardial longitudinal strains at rest and under stress in a population with suspected CAD presenting at chest pain unit. A strong evidence was established that coronary stenosis can impair strain at rest and 2D longitudinal strain can detect CAD. ${ }^{(10,11,12)}$ However, the power of the GLS under stress in detecting the presence or severity of CAD is still uncertain. GLS is reproducible regardless of long term experience in echocardiography. ${ }^{13}$

Visual assessment of WMA under stress showed a weak correlation to the presence of CAD and had no significant correlation with the severity of coronary lesion. We didn't include the assessment of resting WMA in our statistical analysis due to established intermediate sensitivity and specificity and lack of novelty of such evaluation against WMA under stress.

We detected a significant correlation of global longitudinal strain, both at rest and under dobutamine stress, with the occurrence of CAD and with the severity of the coronary lesion. Furthermore, a cut-off value of GLS at rest and under stress was determined based on the number of diseased coronary vessels. In our prospective study, GLS under stress showed higher sensitivity and specificity than both GLS at rest and visual assessment of WMA in dobutamine echocardiography. Few GLS values at rest were lower than $-14 \%$. In such patients we assume an underlying small vessel disease or microvascular dysfunction which couldn't be detected in the coronary angiography. Whereas a concomitant peri- or myocarditis can't be excluded either.

Studies reported that regional 2D strain was significantly lower in the corresponding segments supplied by the stenotic coronary artery and proposed that impaired regional longitudinal strains could help identify which coronary artery is stenotic ${ }^{(14,15)}$. Yet its diagnostic value has not been fully entrenched. $(16,17)$ In our current study we documented a significant correlation of GLS at rest and under stress with both severity of coronary lesion and number of involved coronary vessels. 
The study by dos Santos et al. examined the applicability of left ventricular longitudinal strain at the emergency room. ${ }^{(18)}$ The authors assessed 78 patients with clinically suspected unstable angina pectoris. The presence of severe coronary lesions was confirmed by coronary cineangiography in most of the fifteen patients considered eligible for 2D-STE. Besides, the authors observed that global strain was significantly reduced in patients with severe lesions in any epicardial coronary artery and that the longitudinal strain was significantly reduced in the basal segments of left ventricular inferior and lateral walls of the right and circumflex coronary arteries.

These findings support our current evidence showing that reduced longitudinal strain is correlated with the severity of myocardial ischemia in terms of the number of coronary vessels affected.

Another Swedish study which included 296 consecutive patients with clinically suspected stable angina pectoris and normal left ventricular ejection fraction showed that global longitudinal peak systolic strain assessed at rest is an independent predictor of significant CAD and significantly improves the diagnostic performance of exercise test. Furthermore, 2-dimensional strain echocardiography seemed capable of identifying high-risk patients. ${ }^{(19)}$

In 2018, Scharrenbroich and his coauthors evaluated differences in strain obtained by speckle tracking and left ventricular ejection fraction to predict cardiac events in patients after acute myocardial infarction compared to patients with known coronary artery disease. ${ }^{(20)}$

In CAD patients, ROC analysis demonstrated that the addition of endocardial GCS (global circumferential strain) to baseline characteristics and ejection fraction to a regression model significantly improved the prediction of cardiac events (area under curve $=0.86$, cutoff value: $20 \%$, sensitivity: $79 \%$, specificity: $84 \%$ ).

A cross-sectional study which was performed in Bangladesh included patients with acute coronary syndrome who underwent 3D speckle tracking echocardiography immediately prior to coronary angiography. The patients were categorized upon presence of significant stenosis in the coronary angiography to two groups. ${ }^{(21)}$

All strain parameters were significantly reduced in patient group of significant stenosis. Receiver operating characteristic (ROC) curve analysis demonstrated that GPSLS could effectively detect patients with significant stenosis (area under ROC curve $=0.840,95 \% \mathrm{Cl}=0.735-0.945$ ). GPSLS with a cutoff value of $-13.50 \%$ showed good sensitivity and specificity for predicting significant stenosis (sensitivity $88.9 \%$ and specificity $70.8 \%)$.

In patients without visible regional WMA during pharmacological stress testing and typical angina pectoris symptoms, clinical decision making could be doubtful. In such patients, adding strain measurements with conventional stress echocardiography can provide additional diagnostic value. ${ }^{(22)}$

\section{Limitations}


Heart rate variability played a negative role in strain rate offline analysis. The analysis was aborted and many patients were therefore excluded from the study. Patients with atrial fibrillation were also excluded as the program couldn't overcome the heart rate variability during the study. Moreover, Failure of obtaining a perfect acoustic window during performing the echocardiography remains a critical limitation in markedly obese patients or hyper-inflated thoracic wall. Obtaining sufficient quality images under stress was challenging in some cases. In addition, this research is based on a single center study with relatively limited number of studied objects. The severity of coronary occlusion was determined mostly based on angiography criteria, only 23 patients received further assessment through FFR (fractionated flow reserve) or IFR (instantaneous wave-free ratio).

\section{Conclusion}

Longitudinal strain parameters at rest and under stress could predict the presence of coronary artery disease in patients with stable angina pectoris. They could identify the severity of coronary artery involvement prior to invasive diagnostics. Strain parameters under stress showed higher sensitivity and specificity in comparison to rest parameters in detecting the presence of CAD and in comparison to traditional visual assessment of wall motion abnormalities in stress echocardiography. Measuring global longitudinal strain under stress is highly recommended prior to invasive coronary investigation in patients with stable angina pectoris.

\section{Declarations}

Ethical approval was obtained from the ethical committee prior to recruiting patients in the study. Every patient signed a consent before participating in this work.

All authors have no conflict of interests regarding this research.

Authors of this study approve the publication of this work in "Cardiovascular Ultrasound".

This study was conducted at the cardiology department of Coburg hospital without obtaining external funds. Study materials are available upon request.

The first author is the main and responsible author of this work. All other Authors contributed equally to this research.

The authors would like to thank Prof. Z. Dogas and Ass. Prof. I. Kolcic, University of Split, Croatia for supporting this study

\section{References}

1. Hausmann H, Topp H, Siniawski H, Holz S, and Hetzer R (1997) Decision Making in End-Stage Coronary Artery Disease: Revascularization or Heart Transplantation? Ann Thorac Surg 64:1296- 
1302.

2. Hoffmann $\mathrm{R}$, Lethen $\mathrm{H}$, and Marwick $\mathrm{T}$, et al. Analysis of interinstitutional observer agreement in interpretation of dobutamine stress echocardiograms. J Am Coll Cardiol. 1996; 27:330-6.

3. Picano E, Lattanzi F, and Orlandini A, et al. Stress echocardiography and the human factor: the importance of being expert. J Am Coll Cardiol. 1991; 17:666-9.

4. Franke A, Hoffmann R, Kuhl HP, Lepper W, Breithardt OA, Schormann M et al. Non-contrast second harmonic imaging improves interobserver agreement and accuracy of dobutamine stress echocardiography in patients with impaired image quality. Heart 2000;83:133-40.

5. Hoffmann R, Marwick TH, Poldermans D, Lethen H, Ciani R, and van der Meer P et al. Refinements in stress echocardiographic techniques improve inter-institutional agreement in interpretation of dobutamine stress echocardiograms. Eur Heart J 2002;23:821-9.

6. Stoylen A, Heimdal A, and Bjornstad K, et al. SRI by ultrasonography in the diagnosis of coronary artery disease. J Am Soc Echocardiogr. 2000;13: 1053-1064.

7. Jamal F, Strotmann J, and Weidemann F, et al. Noninvasive quantification of the contractile reserve of stunned myocardium by ultrasonic strain rate and strain. Circulation. 2001;104:1059-1065.

8. Weidemann F, Jamal F, and Sutherland G, et al. Myocardial function defined by strain rate and strain during alterations in inotropic states and heart rate. Am J Physiol. 2002;283:H792-H799.

9. Abraham TP, Belohlavek $\mathrm{M}$, and Thomson $\mathrm{H}$, et al. Time to onset of regional relaxation: feasibility, variability and utility of a novel index of regional myocardial function by SRI. J Am Coll Cardiol. 2002;39:1531-1537.

10. Shimoni S, Gendelman G, and Ayzenberg O, et al.Differential effects of coronary artery stenosis on myocardial function: The value of myocardial strainanalysis for the detection of coronary artery disease.J Am SocEchocardiogr, 2011,24(7):748-757.

11. Montgomery DE, Puthumana JJ, and Fox JM, et al. Global longitudinal strain aids the detection of nonobstructive coronary artery disease in the resting Eur Heart J Cardiovasc Imaging, 2012,13(7):579-587.

12. Choi JO, Cho SW, and Song YB, et al. Longitudinal 2D strain at rest predicts the presence of left main and three vessel coronary artery disease in patients without regional wall motion abnormality. Eur $\mathrm{J}$ Echocardiogr, 2009,10(5):695-701.

13. Karlsen, S., Dahlslett, T., Grenne, B. et al. Global longitudinal strain is a more reproducible measure of left ventricular function than ejection fraction regardless of echocardiographic training. Cardiovasc Ultrasound 17, 18 (2019).

14. Biering-Sorensen T, Hoffmann S, and Mogelvang R, et al.Myocardial strain analysis by 2-dimensional speckletracking echocardiography improves diagnostics ofcoronary artery stenosis in stable angina pectoris. Circ Cardiovasc Imaging, 2014,7(1):58-65.

15. Yang $B$, Daimon $M$, and Ishii $K$, et al. Prediction ofcoronary artery stenosis at rest in patients with normal left ventricular wall motion. Segmentalanalyses using strain imaging diastolic index. IntHeart J, 2013,54(5):266-272. 
16. Stankovic I, Putnikovic B, and Cvjetan R, et al. Visual assessment vs. strain imaging for the detection of critical stenosis of the left anterior descendingcoronary artery in patients without a history ofmyocardial infarction. Eur Heart J Cardiovasclmaging, 2015,16(4):402-409.

17. Hanekom L, Cho GY, and Leano R, et al. Comparisonof two-dimensional speckle and tissue Dopplerstrain measurement during dobutamine stressechocardiography: An angiographic correlation. EurHeart J, 2007,28(14):1765-1772.

18. dos Santos NS, Vilela AA, Barretto RB, Vale MP, Rezende MO, and Ferreira MC, et al. Aplicabilidade do Strain Longitudinal do Ventrículo Esquerdo na Angina Instável. Arq Bras Cardiol. 2018; 110(4):354361.

19. Tor Biering-Sørensen, Soren Hoffmann, Rasmus Mogelvang, , Allan Zeeberg Iversen, Søren Galatius, Thomas Fritz-Hansen, Jan Bech, and Jan Skov Jensen, et al. Myocardial Strain Analysis by 2Dimensional Speckle Tracking Echocardiography Improves Diagnostics of Coronary Artery Stenosis in Stable Angina Pectoris. Circulation: Cardiovascular Imaging. 2014;7:58-65.

20. Scharrenbroich J., Hamada S., and Keszei A., et al. Use of two-dimensional speckle tracking echocardiography to predict cardiac events: Comparison of patients with acute myocardial infarction and chronic coronary artery disease. Clin Cardiol. 2018; 41: 111- 118.

21. Biswas, AK, Haque, T, Banik, D, Choudhury, SR, Khan, SR, and Malik, F-T. et al. Identification of significant coronary artery disease in patients with non-ST segment elevation acute coronary syndrome by myocardial strain analyses using three-dimensional speckle tracking echocardiography. Echocardiography. 2018; 35: 1988- 1996.

22. Giulia Elena Mandoli Maria Concetta Pastore, and Matteo Cameli et al. Speckle tracking stress echocardiography: A valuable diagnostic technique or a burden for everyday practice? Echocardiography. Volume37, Issue12.December 2020. 2123-2129.

\section{Tables}

Table 1. Baseline characteristics based on presence or absence of coronary artery disease (CAD). No: number. \%: percentage in each group (no CAD or CAD group). DM: diabetes mellitus. EF: ejection fraction. GLS: global longitudinal strain. 


\begin{tabular}{|lllc|}
\hline & No CAD & CAD & p-value \\
\hline Age (years) & $65 \pm 12$ & $63 \pm 9$ & 0.519 \\
\hline Men no. & 13 & 41 & 0.05 \\
\hline Hypertension no. (\%) & $27(77.1)$ & $60(82.2)$ & 0.353 \\
\hline DM no. (\%) & $21(60.0)$ & $31(42.5)$ & 0.067 \\
\hline Hyperlipidemia no. (\%) & $12(34.3)$ & $28(38.4)$ & 0.424 \\
\hline EF (\%) & $58.0 \pm 6.4$ & $48.9 \pm 5.9$ & $<0.001$ \\
\hline Troponin (ng/ml) & & & \\
\hline WMA in stress echocardiography (no.) & 77 & $0.081 \pm 0.07$ & 0.315 \\
\hline GLS at rest (\%) & $-18.4 \pm 3.1$ & $-16.9 \pm 2.8$ & $<0.001$ \\
\hline GLS under stress (\%) & & & 0.006 \\
\hline
\end{tabular}

Table 2. Sensitivity, specificity, positive predictive value and negative predictive value of visual wall motion abnormalities (WMA) under stress and strain parameters at rest and under stress in detecting CAD. Values in $\%$, $95 \% \mathrm{Cl}$

\begin{tabular}{|llll|}
\hline & Visual WMA & $\begin{array}{l}\text { Global longitudinal Strain } \\
\text { at rest }\end{array}$ & $\begin{array}{l}\text { Global longitudinal strain } \\
\text { under stress }\end{array}$ \\
\hline Sensitivity & $\begin{array}{l}61.2(42.2- \\
78.2)\end{array}$ & $70.9(51.9-85.7)$ & $74.2(55.3-88.1)$ \\
\hline Specificity & $\begin{array}{l}67.5(55.9- \\
77.8)\end{array}$ & $68.8(57.3-78.9)$ & $76.7(65.5-85.5)$ \\
\hline $\begin{array}{l}\text { Positive predictive } \\
\text { value }\end{array}$ & $\begin{array}{l}44.2(33.9- \\
54.9)\end{array}$ & $47.8(38.0-57.7)$ & $56.1(44.7-66.8)$ \\
\hline $\begin{array}{l}\text { Negative predictive } \\
\text { value }\end{array}$ & $\begin{array}{l}81.2(73.1- \\
87.3)\end{array}$ & $85.5(76.9-91.2)$ & $88.1(80.0-93.1)$ \\
\hline
\end{tabular}

Figures 


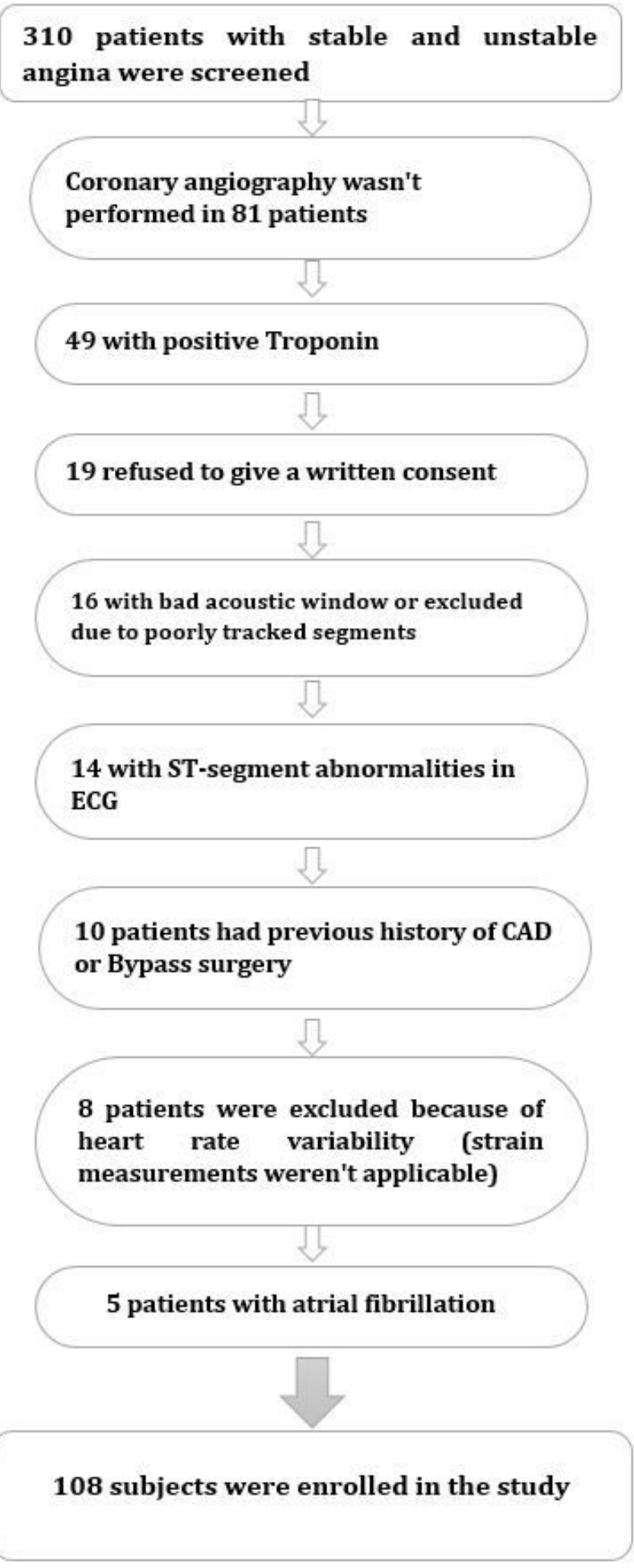

\section{Figure 1}

Flow chart of patients enrolled in the study and reasons for exclusion. 


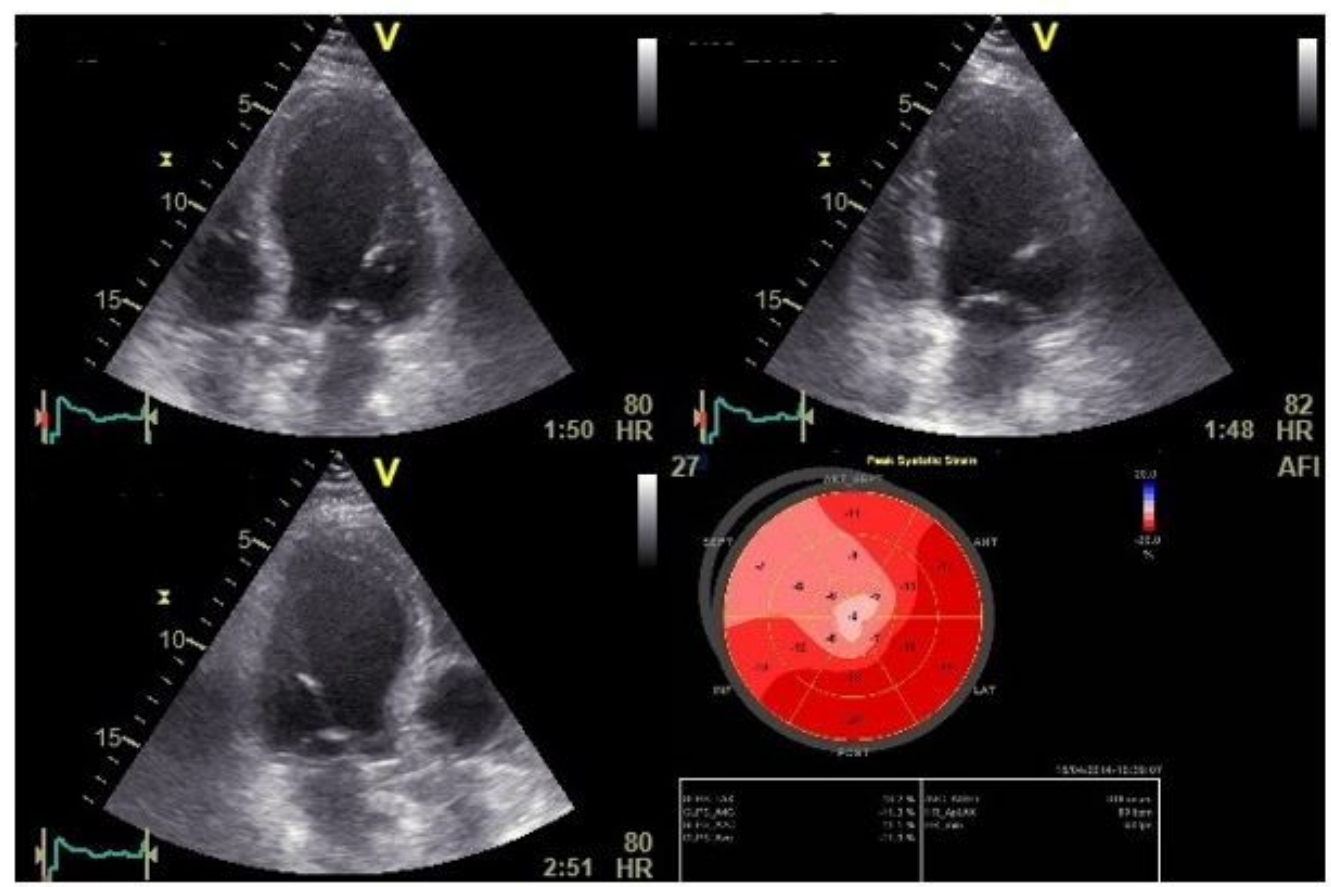

b

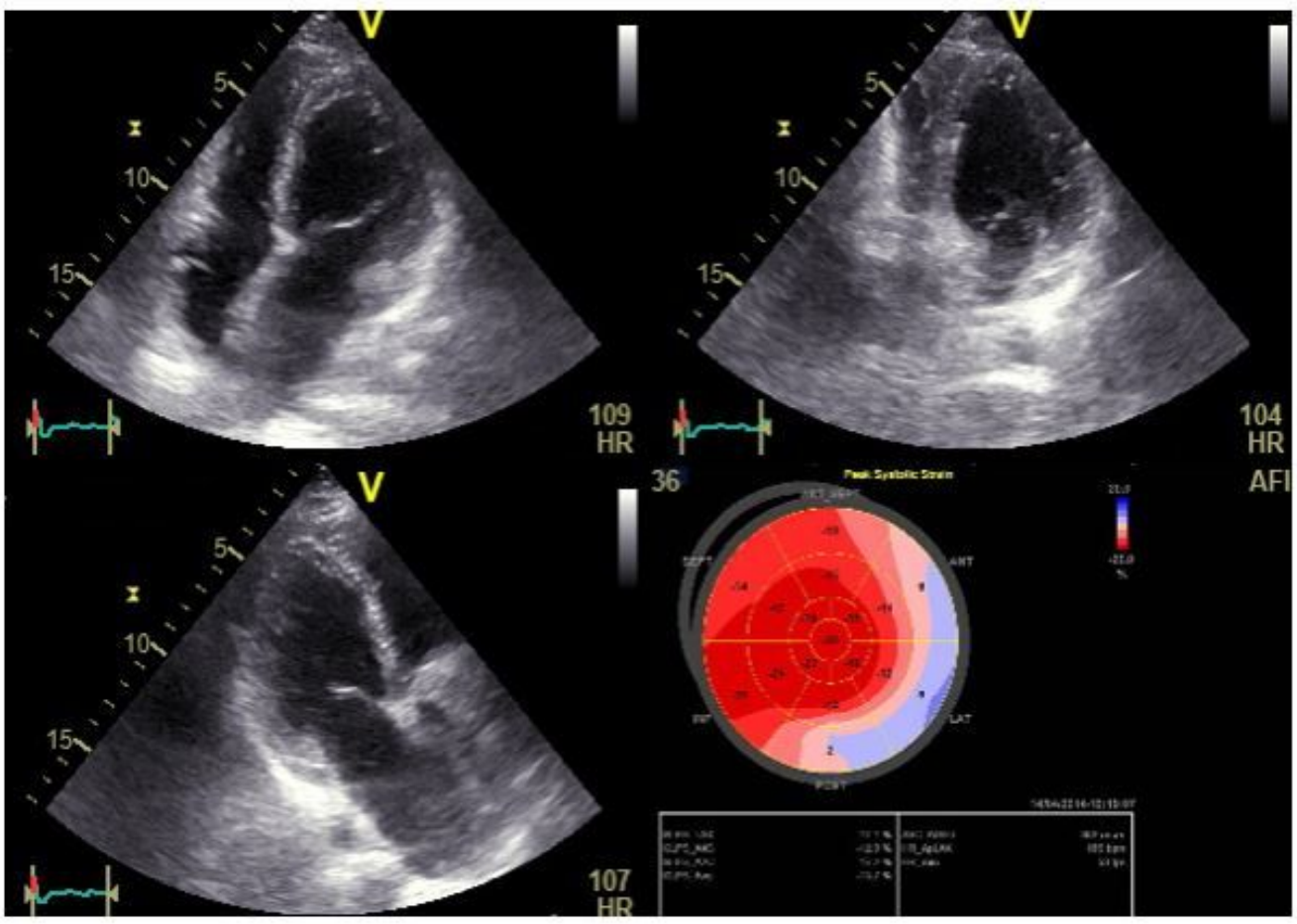

Figure 2

a. Global longitudinal strain (GLS) by speckle tracking at rest showing decreased strain in septal region. b. GLS by speckle tracking under stress (first phase stress at $20 \mathrm{mg} / \mathrm{kg} / \mathrm{min}$ intravenous infusion of dobutamine) of the same patient showing decreased strain distributed over anterior and anterolateral region (significant Left circumflex stenosis was revealed in coronary angiography). 


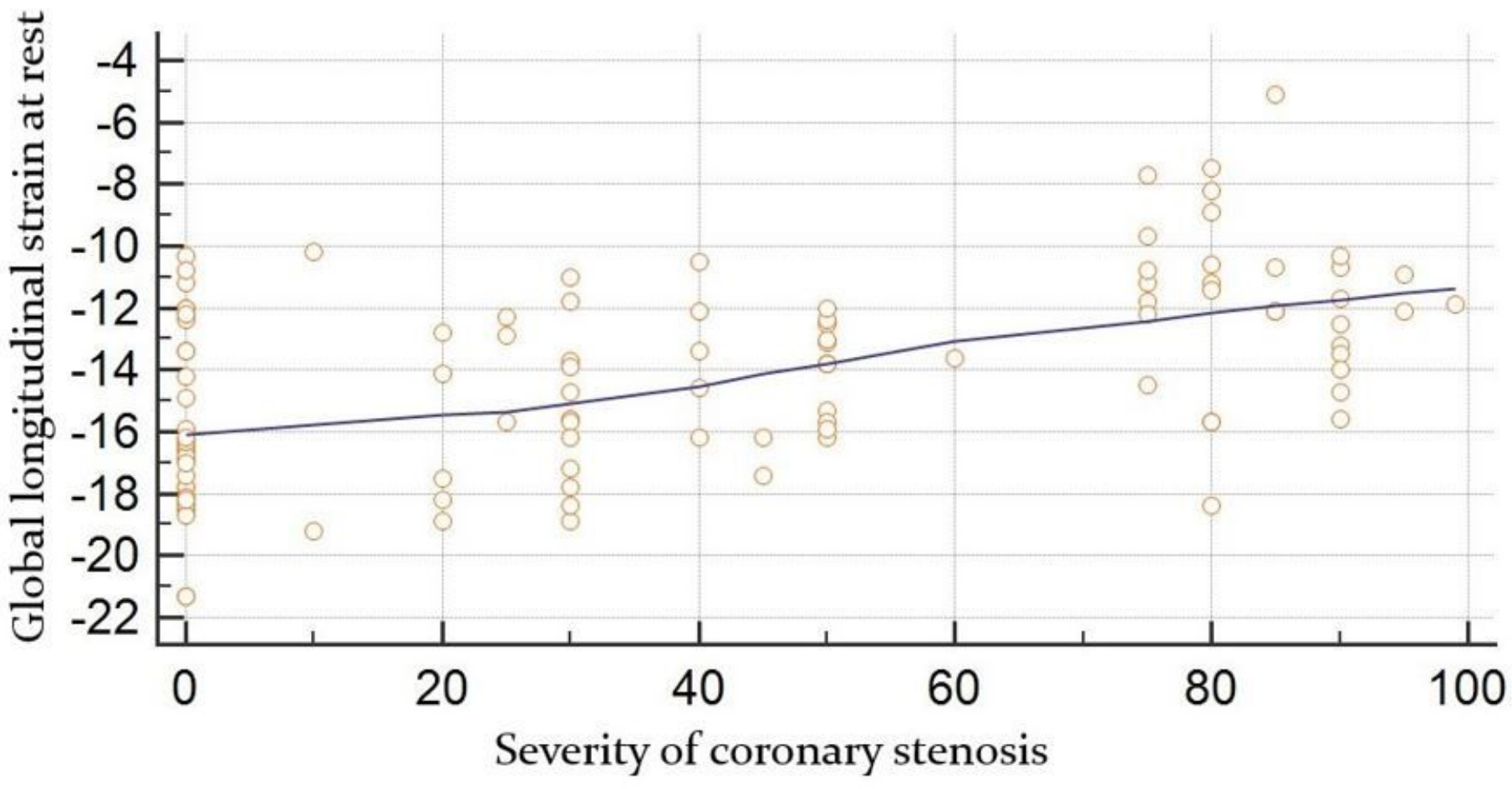

Figure 3

Correlation between global longitudinal strain at rest obtained by speckle tracking (in \%) and the angiographic severity of coronary lesion (in \%).

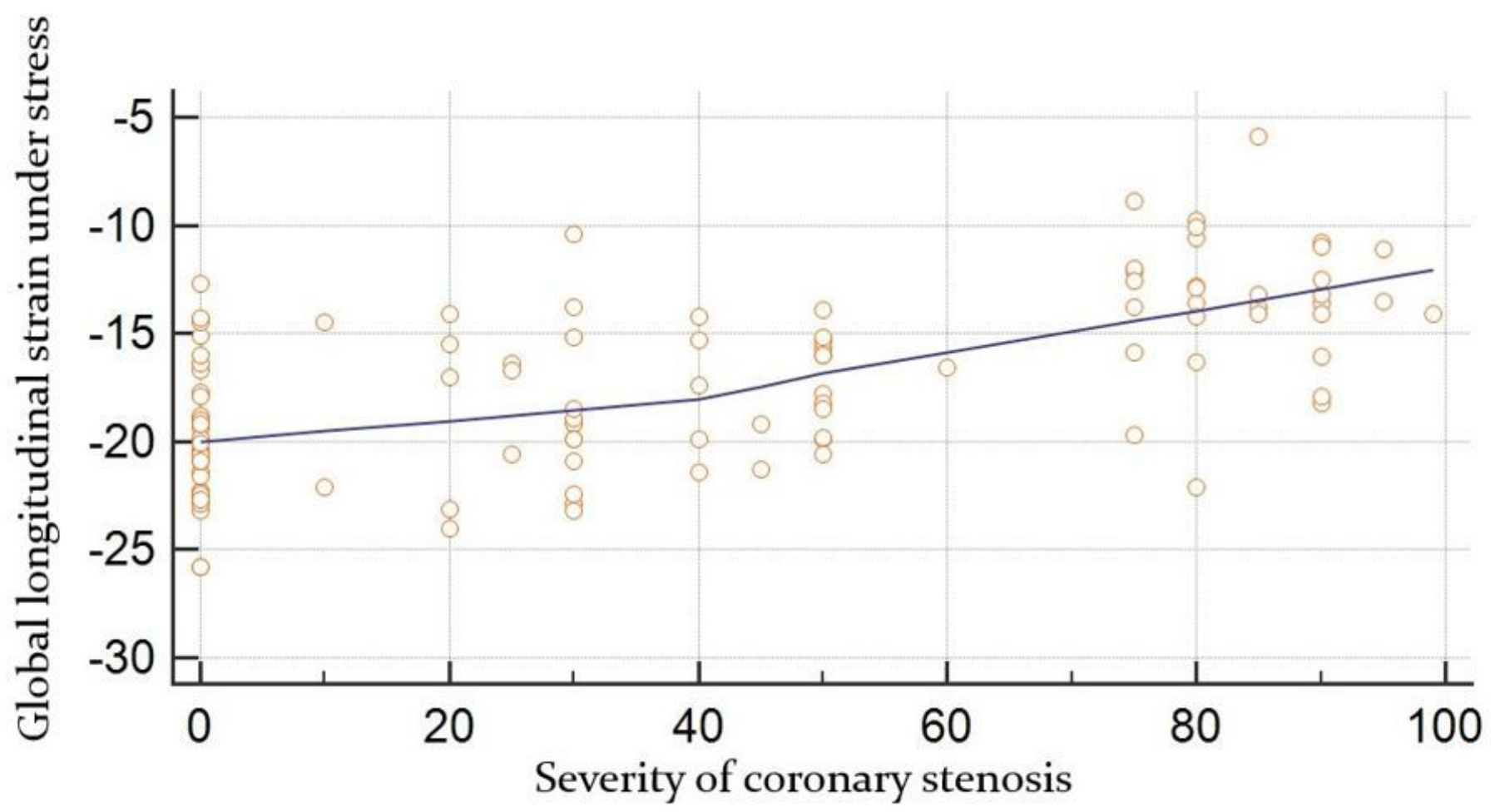

Figure 4 
Correlation between global longitudinal strain under stress obtained by speckle tracking (in \%) and the angiographic severity of coronary lesion (in \%).

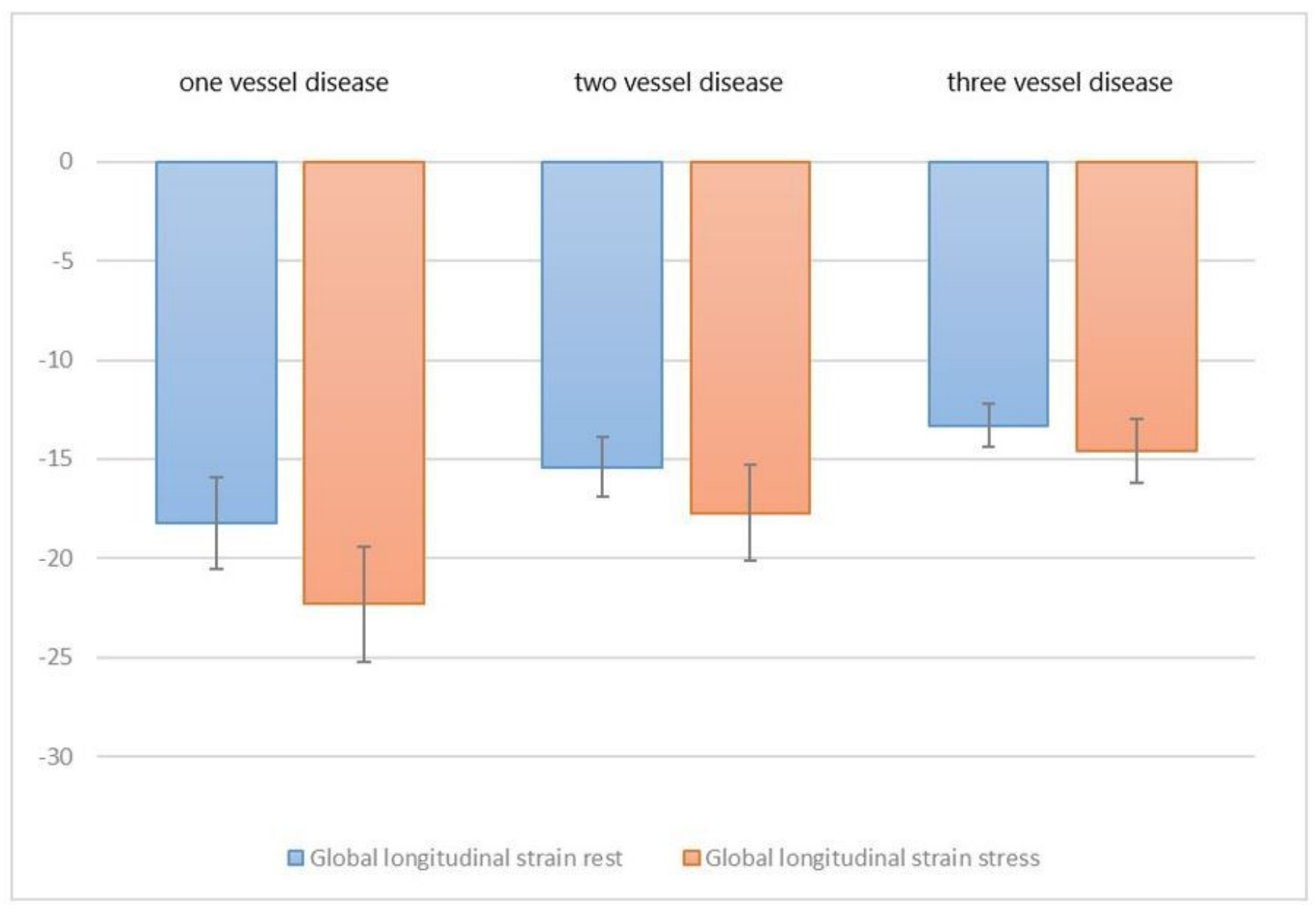

\section{Figure 5}

Mean values of global longitudinal strain at rest and under stress in relation to the number of involved coronary vessels. 


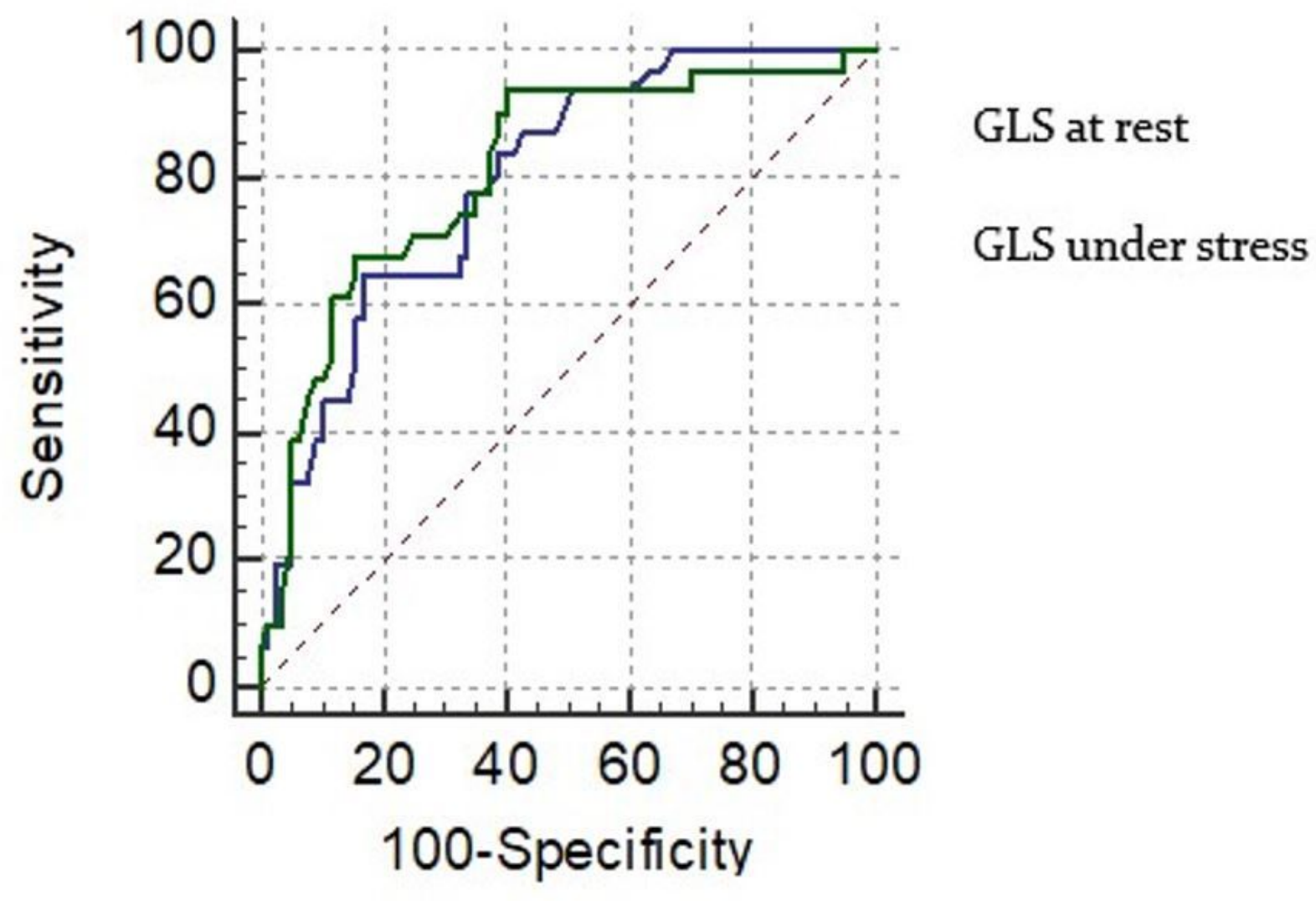

Figure 6

Receiver operating curve for sensitivity and specificity of GLS (global longitudinal strain) at rest and under dobutamine stress in detecting significant coronary artery disease. 